Enferm Bras 2021;20(4):535-48

doi: $10.33233 /$ eb.v20i4.4590

\title{
REVISÃO
}

\section{COVID-19 na assistência ao parto e nascimento}

Liniker Scolfild Rodrigues da Silva*, Tarcísia Domingos de Araújo, M.Sc. ${ }^{* *}$, Jadson Rodrigo de Freitas ${ }^{* * *}$, Cinthia Ferreira Regis ${ }^{* * * *}$, Joana D'Arc Vila Nova Jatobá ${ }^{* * * *}$, Fernanda da Mata Vasconcelos Silva ${ }^{\star \star * \star * *}$

*Enfermeiro, Especialista em Enfermagem Obstétrica na modalidade Residência pela Faculdade de Enfermagem Nossa Senhora das Graças (FENSG)/Universidade de Pernambuco (UPE), Recife, PE, ${ }^{* * E n f e r m e i r a, ~ M e s t r e ~ e m ~ E n f e r m a g e m ~ p e l a ~}$ Universidade de Pernambuco/UPE, Recife, $P E,{ }^{* * *}$ Enfermeiro pelo Centro Universitário Maurício de Nassau (UNINASSAU), Caruaru, PE, ${ }^{* * \star}$ Enfermeira Mestre em Educação em Saúde pela Faculdade Pernambucana de Saúde/FPS, Recife, PE, ${ }^{* * * \star E n f e r m e i r a, ~}$ Doutoranda em Ciências da Saúde pela Faculdade de Medicina do ABC, Recife, PE, ${ }^{* * * * *}$ Enfermeira, Doutoranda em Enfermagem pela Universidade de Pernambuco/UPE, Recife, $P E$

Recebido em 18 de fevereiro de 2021; Aceito em 20 de junho de 2021.

Correspondência: Fernanda da Mata Vasconcelos Silva, Rua Vicente do Rego Monteiro,292 Cordeiro 50630-710 Recife PE

Liniker Scolfild Rodrigues da Silva: liniker_14@hotmail.com

Tarcísia Domingos de Araújo: tarcisiadsouza@gmail.com

Jadson Rodrigo de Freitas: jadson.caruaru@hotmail.com

Cinthia Ferreira Regis: cinthiaregis@outlook.com

Joana D'Arc Vila Nova Jatobá: jdvnj@hotmail.com

Fernanda da Mata Vasconcelos Silva: nandadamata34@gmail.com

\section{Resumo}

Introdução: Gestantes e recém-nascidos são considerados pela Organização Mundial da Saúde como vulneráveis para infecção pelo novo coronavírus. Evidências indicam atenção prioritária a esses grupos uma vez que não existe definição científica sobre as formas de contágio, mecanismo de ação viral e potencial patogênico, incluindo nesse contexto as dúvidas sobre a transmissão vertical e amamentação. Objetivo: Sintetizar as evidências disponíveis na literatura científica acerca dos impactos causados pela 
pandemia da COVID-19 frente a assistência ao parto e nascimento. Métodos: Revisão integrativa da literatura realizada nas bases da Medline/Pubmed, PMC, DOAJ, Medline/BVS, BDEnf, Scielo e Web of Science. Resultados: Dos 54 estudos encontrados, 18 estavam disponíveis na Medline/Pubmed; 16 na PMC; 11 na DOAJ; 7 na Medline/BVS, 2 na BDEnf, 11 na SCOPUS. Após a leitura criteriosa dos artigos, 10 artigos compuseram a amostra. Conclusão: Os artigos explicitaram desenvolvimento de conselhos internacionais para parteiras, outros profissionais de saúde e gerentes de serviços de saúde sobre o cuidado de mulheres e seus bebês durante o parto; adoção de um sistema paternalista de abordagem ao parto somado ao medo das gestantes; esclarecimento às suposições acerca do risco da COVID-19 no terceiro trimestre da gravidez; e estabeleceram um comparativo entre o número de gestantes e não gestantes que testaram positivo identificando de forma precoce de qualquer impacto psicológico que ocorra na gestação.

Palavras-chave: infecções por coronavirus; saúde da mulher; parto.

\section{Abstract}

\section{COVID-19 pandemic in delivery and birth assistance}

Introduction: Pregnant women and newborns are considered by the World Health Organization to be vulnerable to infection with the new coronavirus. Evidence indicates priority attention to these groups since there is no scientific definition on the forms of contagion, mechanism of viral action and pathogenic potential, including in this context doubts about vertical transmission and breastfeeding. Objective: To synthesize the evidence available in the scientific literature about the impacts caused by the pandemic of COVID-19 in relation to childbirth and birth assistance. Methods: Integrative literature review carried out on the database of Medline/Pubmed, PMC, DOAJ, Medline/BVS, BDEnf, Scielo and Web of Science. Results: Of the 54 studies found, 18 were available at Medline/Pubmed; 16 at PMC; 11 at DOAJ; 7 at Medline/BVS, 2 at BDEnf, 11 at SCOPUS. After careful reading of the articles, 10 articles made up the sample. Conclusion: The articles made explicit the development of international advice for midwives, other health professionals and health service managers on the care of women and their babies during childbirth; adoption of a paternalistic system of approach to childbirth added to the fear of pregnant women; clarification of assumptions about COVID-19 risk in the third trimester of pregnancy; and established a comparison between the number of pregnant women and non-pregnant women who tested positive, identifying early any psychological impact that occurs during pregnancy.

Keywords: coronavirus infections; women's health; parturition. 


\section{Resumen}

\section{COVID-19 en la asistencia al parto y nacimiento}

Introducción: La Organización Mundial de la Salud considera que las mujeres embarazadas y los recién nacidos son vulnerables a la infección por el nuevo coronavirus. La evidencia indica una atención prioritaria a estos grupos ya que no existe una definición científica sobre las formas de contagio, mecanismo de acción viral y potencial patogénico, incluyendo en este contexto dudas sobre la transmisión vertical y la lactancia materna. Objetivo: Sintetizar la evidencia disponible en la literatura científica sobre los impactos provocados por la pandemia de COVID-19 en relación con el parto y la asistencia al parto. Métodos: Revisión integrativa de la literatura realizada sobre las bases de Medline/PubMed, PMC, DOAJ, Medline/BVS, BDEnf, Scielo y Web of Science. Resultados: De los 54 estudios encontrados, 18 estaban disponibles en Medline/PubMed; 16 en PMC; 11 en DOAJ; 7 en Medline/BVS, 2 en BDEnf, 11 en SCOPUS. Después de leer atentamente los artículos, 10 artículos conformaron la muestra. Conclusión: Los artículos explicitaron el desarrollo de asesoramiento internacional para parteras, otros profesionales de la salud y administradores de servicios de salud sobre el cuidado de las mujeres y sus bebés durante el parto; la adopción de un sistema paternalista de abordaje del parto, sumado al miedo de las mujeres embarazadas; aclaración de las suposiciones sobre el riesgo de COVID-19 en el tercer trimestre del embarazo; y estableció una comparación entre el número de mujeres embarazadas y no embarazadas que dieron positivo, identificando temprano cualquier impacto psicológico que ocurra durante el embarazo.

Palabras-clave: infecciones por coronavirus; salud de la mujer; parto.

\section{Introdução}

A infecção pelo vírus COVID-19, que teve início na cidade de Wuhan, na China, se alastrou rapidamente por todos os países do mundo, ganhando proporção de pandemia [1]. Desde então, as autoridades sanitárias e sociedades científicas nacionais e internacionais têm divulgado informações e realizando campanha de conscientização para orientar a comunidade e os profissionais de saúde sobre as medidas e ações estratégicas voltadas à prevenção da COVID-19, que atualmente é considerada uma doença de notificação compulsória [2].

Idosos, profissionais da saúde, pessoas imunossuprimidas, com doenças crônicas, gestantes e recém-nascidos são considerados, pela Organização Mundial da Saúde (OMS), como vulneráveis para infecção pelo novo coronavírus [1]. Evidências disponíveis indicam atenção prioritária a esses grupos, uma vez que não existe definição 
científica sobre as formas de contágio, mecanismo de ação viral e potencial patogênico, incluindo nesse contexto as dúvidas sobre a transmissão vertical e amamentação [4].

No caso das gestantes, a orientação em saúde deve ser realizada de acordo com cada estágio do ciclo gravídico-puerperal [5,6]. A gestação é um momento da vida da mulher influenciado por inúmeras mudanças fisiológicas e comportamentais. As alterações se acentuam mediante as incertezas advindas das variantes clínicas que as mesmas apresentam ao serem infectadas pelo coronavírus. Tal perspectiva gera nessas mulheres um sentimento de medo e insegurança que impacta no processo gestacional e pós-parto [5].

Inúmeras incertezas permeiam a história natural e o manejo clínico da COVID19 durante a gestação e os primeiros meses de vida. Estudos estão sendo realizados para identificar vacinas e tratamentos específicos. No entanto, o ciclo da vida permanece contínuo e com ele os nascimentos durante esse período, o que aumenta ainda mais as dúvidas relacionadas aos cuidados a serem adotados na vigência da pandemia [1,2]. A grande preocupação em saúde é com o desenvolvimento das formas graves da doença e da necessidade de suporte assistencial em unidades de terapia semi-intensiva ou intensiva, visto que estes setores estão operando além de suas capacidades [7].

Os serviços de atenção obstétrica e neonatal são considerados essenciais e, portanto, a infecção pela COVID-19 tem direcionado a atenção de pesquisadores para a criação de estratégias de prevenção e gestão clínica da infecção COVID-19 em gestantes e recém-nascidos. Torna-se indispensável, para tanto, que profissionais que atuam no cuidado estejam atualizados e treinados para tomada de decisões daqueles com suspeita ou infecção confirmada por SARS-CoV-2 [8].

Diante do exposto, o estudo tem por objetivo sintetizar as evidências disponíveis na literatura científica acerca dos impactos causados pela pandemia do COVID-19 frente a assistência ao parto e nascimento.

\section{Material e métodos}

Trata-se de uma revisão integrativa da literatura desenvolvida a partir de critérios metodológicos que percorreram as seguintes etapas: elaboração da questão norteadora e objetivo do estudo; definição de critérios de inclusão e exclusão das produções científicas; busca de estudos científicos nas bases de dados e bibliotecas virtuais; análise e categorização das produções encontradas; resultados e discussão dos achados [9]. 
Para o levantamento da questão norteadora, utilizou-se PICo (P: Gestantes; I: Impacto da pandemia do COVID-19 na saúde; Co: Assistência de qualidade ao parto e nascimento [10]. Desta forma, definiu-se a seguinte questão norteadora [7] da pesquisa: "Quais os impactos causados pela pandemia da COVID-19 frente a assistência ao parto e nascimento?".

Para seleção dos artigos, utilizaram-se como critérios de inclusão: ser artigo científico disponível na íntegra, com delimitação atemporal, publicado em português, inglês ou espanhol que respondessem ao objetivo do estudo e que possibilitasse o acesso pelo Virtual Private Network (VPN) da Universidade de São Paulo (USP). Foram excluídas as literaturas cinzas, estudos de reflexão, bem como publicações repetidas de estudos em mais de uma base de dados ou biblioteca virtual e os artigos que não responderam à questão norteadora do estudo. Justifica-se o estudo atemporal uma vez que a identificação do vírus é recente (2019).

O levantamento dos dados ocorreu durante o mês de janeiro de 2021 nas seguintes bases de dados e bibliotecas virtuais: Banco de Dados em Enfermagem (BDEnf), Pubmed Central (PMC), Medical Literature Analysis and Retrieval System Online (Medline)/PubMed, Directory of Open Access Journals (DOAJ), Medline/Biblioteca Virtual em Saúde (BVS), Scientific Electronic Library Online (Scielo), Web of Science e SCOPUS. Elegendo estas bases de dados e bibliotecas por entender que atingem a literatura publicada, como também referências técnico-científicas brasileiras em saúde pública e coletiva.

Buscaram-se os artigos a partir dos Descritores em Ciências da Saúde (DeCS): "Infecções por Coronavirus", "Saúde da Mulher", "Parto". Utilizaram-se os respectivos termos provenientes do Medical Subject Headings (MeSH): "Coronavirus Infections", "Women's Health", "Parturition". Combinados com operador booleano AND e OR, efetuando a busca conjunta e individualmente para que possíveis diferenças fossem corrigidas.

A seleção dos estudos baseou-se no Preferred Reporting Items for Systematic Review and Meta-Analyse (PRISMA) [11]. A princípio eliminaram-se por meio da leitura de títulos e resumos, estudos duplicados. Destes pré-selecionados, realizou-se leitura na íntegra, a fim de verificar os que respondessem à questão norteadora/ de pesquisa. Construiu-se então a amostra final com estudos pertinentes aos critérios préestabelecidos (Figura 1). 


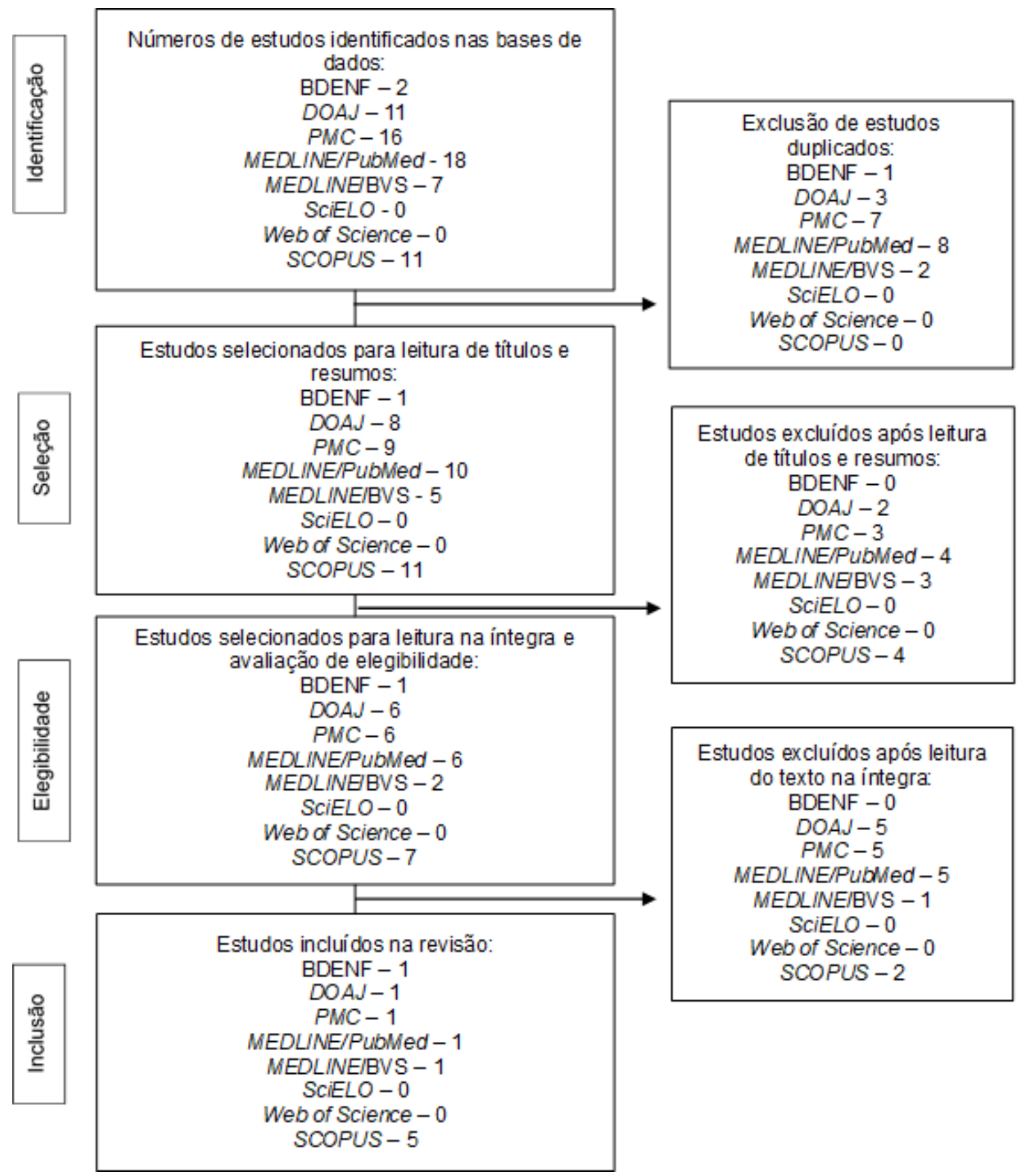

Figura 1 - Fluxograma da seleção dos estudos segundo o Preferred Reporting Items for Systematic Reviews and Meta-Analyses (PRISMA, 2015), Recife, PE, Brasil, 2021

O nível de evidência dos estudos selecionados foi determinado de acordo com a Agency for Healthcare Research and Quality (AHRQ) [12]. Nível I - Metanálise de múltiplos estudos controlados; Nível II - Estudos individuais com delineamento experimental; Nível III - Estudo com delineamento quase-experimental como estudo sem randomização com grupo único pré e pós-teste, séries temporais ou caso-controle; Nível IV- Estudo com delineamento não-experimental como pesquisa descritiva correlacional e qualitativa ou estudos de caso; Nível V - Relatório de casos ou dado obtido de forma sistemática, de qualidade verificável ou dados de avaliação de 
programas; e por fim o Nível VI - Opiniões de autoridades respeitáveis baseada na competência clínica ou opinião de comitês de especialistas, incluindo interpretações de informações não baseadas em pesquisas.

Visando um melhor entendimento das publicações selecionadas nesta revisão, organizaram-se os dados apresentando-os em figuras e tabelas, expostos de forma descritiva.

Resultados e discussão

Tabela I - Resultados encontrados nos estudos de acordo com título, base de dados, autores, ano de publicação, delineamento, local e idioma, Recife, PE, Brasil, 2021

\begin{tabular}{|c|c|c|c|c|}
\hline & Título/Base de dados & Autor/Ano & Delineamento & $\begin{array}{l}\text { Local/ } \\
\text { Idioma }\end{array}$ \\
\hline 1 & $\begin{array}{l}\text { Los derechos de las mujeres en el } \\
\text { parto deben ser respetados durante la } \\
\text { pandemia de Coronavirus. / BDEnf } \\
\text { [13] }\end{array}$ & $\begin{array}{l}\text { International } \\
\text { Confederation of } \\
\text { Midwives (2020) }\end{array}$ & $\begin{array}{l}\text { Estudo de } \\
\text { rastreamento }\end{array}$ & $\begin{array}{l}\text { Estados } \\
\text { Unidos / } \\
\text { Inglês }\end{array}$ \\
\hline 2 & $\begin{array}{l}\text { Fear of childbirth in time of the new } \\
\text { coronavirus pandemic. I } \\
\text { Medline/Pubmed [14] }\end{array}$ & $\begin{array}{l}\text { Souto SPA, } \\
\text { Albuquerque RS, } \\
\text { Prata AP (2020) }\end{array}$ & $\begin{array}{l}\text { Estudo } \\
\text { descritivo }\end{array}$ & $\begin{array}{l}\text { Brasil / } \\
\text { Português }\end{array}$ \\
\hline 3 & $\begin{array}{l}\text { Analysis of maternal coronavirus } \\
\text { infections and neonates born to } \\
\text { mothers with } 2019-n C o V ; \text { a } \\
\text { Systematic Review / PMC [7] }\end{array}$ & $\begin{array}{l}\text { Muhidin S, Behboodi } \\
\text { Moghadam Z, } \\
\text { Vizheh M (2020) }\end{array}$ & $\begin{array}{l}\text { Estudo } \\
\text { descritivo }\end{array}$ & $\begin{array}{l}\text { Estados } \\
\text { Unidos / } \\
\text { Inglês }\end{array}$ \\
\hline 4 & $\begin{array}{l}\text { Post lockdown COVID-19 } \\
\text { seroprevalence and circulation at the } \\
\text { time of delivery in Francel DOAJ [15] }\end{array}$ & $\begin{array}{l}\text { Mattern J et al. } \\
(2020)\end{array}$ & $\begin{array}{l}\text { Estudo } \\
\text { descritivo }\end{array}$ & $\begin{array}{l}\text { Estados } \\
\text { Unidos I } \\
\text { Inglês }\end{array}$ \\
\hline 5 & $\begin{array}{l}\text { Psychosocial factors associated with } \\
\text { postpartum psychological distress } \\
\text { during the COVID-19 pandemic: a } \\
\text { cross-sectional study. / M edline/BVS } \\
\text { [16] }\end{array}$ & $\begin{array}{l}\text { Ostacoli L et al. } \\
(2020)\end{array}$ & $\begin{array}{l}\text { Estudo } \\
\text { descritivo }\end{array}$ & $\begin{array}{l}\text { Itália / } \\
\text { Inglês }\end{array}$ \\
\hline 6 & $\begin{array}{l}\text { Prevention and practice during the } \\
\text { COVID-19 emergency declaration } \\
\text { period in Japanese } \\
\text { obstetrical/gynecological facilities. / } \\
\text { SCOPUS [17] }\end{array}$ & $\begin{array}{l}\text { Komatsu H et al. } \\
(2020)\end{array}$ & $\begin{array}{l}\text { Estudo } \\
\text { qualitativo }\end{array}$ & $\begin{array}{l}\text { Japão/ } \\
\text { Inglês }\end{array}$ \\
\hline 7 & $\begin{array}{l}\text { The psychological experience of } \\
\text { obstetric patients and health care } \\
\text { workers after implementation of } \\
\text { universal SARS-CoV-2 Testing / } \\
\text { SCOPUS [18] }\end{array}$ & $\begin{array}{l}\text { Bender WR et al. } \\
(2020)\end{array}$ & $\begin{array}{l}\text { Estudo } \\
\text { descritivo }\end{array}$ & $\begin{array}{l}\text { Estados } \\
\text { Unidos / } \\
\text { Inglês }\end{array}$ \\
\hline 8 & $\begin{array}{l}\text { Perinatal distress during COVID-19: } \\
\text { Thematic analy sis of an online } \\
\text { parenting forum / SCOPUS [19] }\end{array}$ & $\begin{array}{l}\text { Chivers BR et al. } \\
(2020)\end{array}$ & $\begin{array}{l}\text { Estuo } \\
\text { descritivo }\end{array}$ & $\begin{array}{l}\text { Austrália / } \\
\text { Inglês }\end{array}$ \\
\hline 9 & $\begin{array}{l}\text { Current state of knowledge about } \\
\text { SARS-CoV-2 and COVID-19 disease } \\
\text { in pregnant women. / SCOPUS [20] }\end{array}$ & $\begin{array}{l}\text { Gujski M, Humeniuk } \\
\text { E, Bojar I (2020) }\end{array}$ & $\begin{array}{l}\text { Estudo } \\
\text { descritivo }\end{array}$ & $\begin{array}{l}\text { Polônia / } \\
\text { Inglês }\end{array}$ \\
\hline 10 & $\begin{array}{l}\text { Fear of childbirth in time of the new } \\
\text { coronavirus pandemic/ SCOPUS [21] }\end{array}$ & $\begin{array}{l}\text { Souto SPAD, } \\
\text { Albuquerque RS, } \\
\text { Prata AP (2020) }\end{array}$ & $\begin{array}{l}\text { Estudo } \\
\text { descritivo }\end{array}$ & $\begin{array}{l}\text { Portugal / } \\
\text { Inglês }\end{array}$ \\
\hline
\end{tabular}

Dados da pesquisa, 2021.

Dos artigos encontrados, após a leitura permaneceram os que atendiam aos critérios estabelecidos para inclusão e exclusão descritos em material e métodos. 
Na tabela I, os estudos estão dispostos evidenciando seus títulos, autores, anos de publicação, delineamento, local e idioma. A maioria dos artigos eram internacionais ( $n=8$ ), publicados em inglês, no ano 2020. Um artigo era nacional e publicado em português.

Após a leitura dos artigos selecionados manualmente, os estudos foram categorizados em recortes temáticos, classificando o conhecimento produzido sobre 0 tema, em níveis de evidência, majoritariamente nível IV- Estudo com delineamento nãoexperimental como pesquisa descritiva correlacional e qualitativa ou estudos de caso [9].

Os principais achados, dispostos nos objetivos e conclusões, estão diretamente associados ao impacto da pandemia da COVID-19 frente a assistência ao parto e nascimento, como exposto na tabela II.

Tabela II - Resultados encontrados nos estudos de acordo com os níveis de evidências, objetivos e conclusões, Recife, PE, Brasil, 2021 (ver PDF anexo)

Não há evidências científicas que confirmem maior acometimento de infecção pela COVID-19 em gestantes, comparado à população no geral, diferente do comportamento clínico apresentado pelo grupo em outras infecções virais respiratórias como H1N1, MERS-CoV e SARS-CoV. Os artigos encontrados levantaram evidências dos impactos causados pela pandemia frente a assistência ao parto e nascimento, nas dimensões físicas, emocionais e psicológicas [22,23].

Diversos estudos realizados por enfermeiros averiguaram acerca dos impactos gerados nos cuidados realizados a gestantes e puérperas que testaram positivo para COVID-19. Segundo um consenso emitido pela Associação Médica Chinesa, a infecção por COVID-19 nem sempre indica necessidade de interrupção da gravidez nem antecipação do parto, a variação ocorre considerando, especialmente, o andamento da doença materna, idade gestacional e situação intrauterina do feto. Na maioria das vezes, a transmissão transplacentária é menos provável, visto que o vírus ainda não foi identificado no líquido amniótico, placenta e leite materno das mães ou nas secreções nasais dos seus neonatos, porém, ainda se faz necessário manter um alto nível de vigilância do recém-nascido durante os 14-21 dias após o nascimento [24,25].

Contudo, a infecção neonatal pode acontecer depois do parto através da inalação do SARS-CoV-2 presentes nos aerossóis gerados pela tosse da mãe, parentes ou profissionais de saúde. Portanto, no domicílio, recomenda-se evitar o contato do bebê com pessoas de alto risco para contaminação, como as que apresentam febre ou sintomas respiratórios; evitar sair e proibir a visita de parentes e amigos, e quando estas 
acontecerem o visitante deve usar equipamentos de proteção apropriados; limitar a quantidade de cuidadores; prover boa ventilação, higiene frequente das mãos ao cuidar do bebê; e desinfecção diária dos utensílios dos recém-nascidos [3,26].

Artigos explicitaram desenvolvimento de conselhos internacionais para parteiras, outros profissionais de saúde e gerentes de serviços de saúde sobre o cuidado de mulheres e seus bebês durante o parto; adoção de um sistema paternalista de abordagem ao parto somado ao medo das gestantes; esclarecimento às suposições acerca do risco da COVID-19 no terceiro trimestre da gravidez; e estabeleceram um comparativo entre o número de gestantes e não gestantes que testaram positivo identificando de forma precoce de qualquer impacto psicológico que ocorra na gestação [7,13-16].

Nesse cenário, é de grande importância o papel dos profissionais de enfermagem tanto na atenção Primária à Saúde, durante a consulta do pré-natal ou puerperal, quanto na atenção hospitalar. Vale recordar que, no espaço da atenção primaria, além do recomendado para o atendimento pré-natal, ainda devem estar inclusos orientações acerca dos cuidados à saúde da gestante, desmistificação de algumas ideias preconcebidas, visando a promoção da saúde mental das pacientes que neste momento se encontram cheias de medos e incertezas, e medidas preventivas contra a COVID-19 para promover sua saúde física, como a higiene das mãos e das superfícies, o distanciamento social e o uso e confecção de máscaras. Tais cuidados podem acontecer em inúmeros ambientes, como em grupos de gestantes e na sala de espera [6].

A prestação de cuidados de maneira equivocada no período neonatal pode acarretar serias consequências à saúde do recém-nascido, atingindo sua sobrevivência e o desenvolvimento infantil saudável. Por isso, é imprescindível que os enfermeiros se capacitem por meio de práticas assistenciais e orientações atuais buscando a garantia de cuidados pós-natais seguros e de qualidade, sempre mantendo a valorização do contexto da família e da comunidade [25].

Contudo, as evidências cientificas que tratam sobre esse assunto ainda apresentam algumas fragilidades e lacunas como: unicêntricos, diferentes sistemas de comparação, tamanho pequeno da amostra e falta de randomização.

\section{Conclusão}

A presente revisão proporcionou a identificação de alguns impactos causados pela pandemia da COVID-19 frente a assistência ao parto e ao nascimento. Aspectos causadores de grande preocupação, uma vez que afetam negativamente o bem-estar 
físico, mental e emocional, durante um período que deveria ser só de felicidade, adicionando medos e incertezas. Neste ponto de vista, necessita-se que sejam tomadas medidas que busquem a promoção da atenção à saúde da mulher gestante, em sala de parto e pós-parto, e ao seu recém-nascido, e ainda a redução do impacto negativo, além da diminuição dos índices de morbidade e mortalidade. No entanto, há escassez de estudos que dão a verdadeira importância a saúde da mulher, principalmente gestante, e ao seu recém-nascido em meio a pandemia do COVID-19.

Apesar da literatura que foi investigada sugerir alguns impactos, não há números exatos sobre morbimortalidade materno-infantil, e existe informação de que o país que concentra maior número de mortes de gestantes é o Brasil. Diante disso, percebe-se a necessidade de mais estudos e contribuições científicas com este foco, para que ocorra discussão englobando todos os profissionais, compreendendo uma visão integral da atenção à saúde, buscando o bem-estar como um todo das gestantes e puérperas, e seus recém-nascidos. Portanto, esta revisão tem a finalidade de contribuir com informações para o desenvolvimento de outros estudos, com qualidade metodológica, para que se produzam diretrizes que orientem atividades de prática clínica.

\section{Referências}

1. McIntosh K, Hirsch MS, Bloom A. Coronavirus Disease 2019 (COVID-19): epidemiology, virology, clinical features, diagnosis, and prevention. [Internet] 2020. [cited 2021 Aug 24]. Available from: https://www.uptodate.com/contents/coronavirusdisease-2019-covid-19-epidemiology-virology-clinical-features-diagnosis-andprevention

2. Adams JG, Walls RM. Supporting the health care workforce during the covid-19 global epidemic. JAMA 2020;323(15):1439-40. doi: 10.1001/jama.2020.3972

3. Schwartz DA, Graham AL. Potential maternal and infant outcomes from coronavirus 2019-nCoV (SARS-CoV-2) infecting pregnant women: lessons from SARS, MERS, and other human coronavirus infections. Viruses 2020;12(2):194. doi: 10.3390/v12020194

4. Chen D, Yang H, Cao Y, Cheng W, Duan T, Fan C, et al. Expert consensus for managing pregnant women and neonates born to mothers with suspected or confirmed novel coronavirus (COVID-19) infection. Int J Gynaecol Obstet 2020;149(2):130-6. doi: 10.1002/ijgo.13146

5. Alfaraj SH, Al-Tawfiq JA, Memish ZA. Middle East Respiratory Syndrome Coronavirus (MERS-CoV) infection during pregnancy: Report of two cases \& review of the literature. J Microbiol Immunol Infect 2019;52(3):501-3. doi: 10.1016\%2Fj.jmii.2018.04.005

6. Estrela FM, Silva KKA, Cruz MA, Gomes NP. Gestantes no contexto da pandemia da Covid-19: reflexões e desafios. Physis 2020;30(2):e300215. doi: 10.1590/s010373312020300215 
7. Muhidin S, Behboodi Moghadam Z, Vizheh M. Analysis of maternal coronavirus infections and neonates born to mothers with 2019-nCoV; a Systematic Review. Arch Acad Emerg Med [Internet]. 2020 [cited 2021 Aug 24];8(1):e49. Available from: https://www.ncbi.nlm.nih.gov/pmc/articles/PMC7211430/

8. Araújo WCO. Recuperação da informação em saúde: construção, modelos e estratégias. ConCl: Conv Cienc Inform 2020;3(2):100-134. doi: 10.33467/conci.v3i2.13447

9. Mendes KDS, Silveira RCCP, Galvão CM. Revisão integrativa: método de pesquisa para a incorporação de evidências na saúde e na enfermagem. Texto Contexto Enferm 2008;17(4):758-64. doi: 10.1590/S0104-07072008000400018

10. Santos MARC, Galvão MGA. A elaboração da pergunta adequada de pesquisa. Resid Pediatr [Internet]. 2014 [cited 2021 Aug 24];4(2):53-6. Available from: http://residenciapediatrica.com.br/detalhes/105/a-elaboracao-da-pergunta-adequadade-pesquisa

11. Moher D, Liberati A, Tetzlaff J, Altman DG. The PRISMA Group. Preferred Reporting Items for Systematic Reviews and Meta-Analyses: The PRISMA Statement. Epidemiol Serv Saúde 2015;24(2):335-42. doi: 10.5123/S1679-49742015000200017

12. Jeanne-Marie Guise MD. AHRQ Series on improving translation of evidence: progress and promise in supporting learning health systems. The Joint Commission Journal on Quality and Patient Safety 2020;46:51-2. doi: 10.1016/j.jcjq.2019.10.008

13. International Confederation of Midwives. Los derechos de las mujeres en el parto deben ser respetados durante la pandemia de Coronavirus. Netherlands; ICM; 2020. 4 p.

14. Souto SPA, Albuquerque RS, Prata AP. Fear of childbirth in time of the new coronavirus pandemic. Rev Bras Enferm 2020;73(Suppl 2):e20200551. doi: 10.1590/0034-71672020-0551

15. Mattern J, Vauloup-Fellous C, Zakaria H, Benachi A, Carrara J, Letourneau A, et al. Post lockdown COVID-19 seroprevalence and circulation at the time of delivery, France. PLoS ONE, 2020;15(10):e0240782. doi: 10.1371/journal.pone.0240782

16. Ostacoli L, Cosma S, Bevilacqua F, Berchialla P, Bovetti M, Carosso AR, et al. Psychosocial factors associated with postpartum psychological distress during the Covid-19 pandemic: a cross-sectional study. BMC Pregnancy Childbirth 2020;20(1): 703. doi: 10.1186/s12884-020-03399-5

17. Komatsu H, Banno K, Yanaihara N, Kimura T. Prevention and practice during the COVID-19 emergency declaration period in Japanese obstetrical/gynecological facilities. J Obs Gynaecol Res 2020;46(11):2237-41. doi: 10.1111/jog.14432

18. Bender WR, Srinivas S, Coutifaris P, Acker A, Hirshberg A. The psychological experience of obstetric patients and health care workers after implementation of universal SARS-CoV-2 testing. Am J Perinatol 2020;37(12):1271-9. doi: 10.1055/s0040-1715505 
19. Chivers BR, Garad RM, A Boyle J, Skouteris H, Teede HJ, Harrison CL. Perinatal distress during COVID-19: thematic analysis of an online parenting forum. J Med Internet Res 2020;22(9):22002. doi: 10.2196/22002

20. Gujski M, Humeniuk E, Bojar I. Current state of knowledge about SARS-CoV-2 and COVID-19 disease in pregnant women. Med Sci Monit 2020;26:9247251. doi: $10.12659 / \mathrm{msm} .924725$

21. Souto SPA, Albuquerque RS, Prata AP. Fear of childbirth in time of the new coronavirus pandemic. Rev Bras Enferm 2020;73(2):20200551. doi: 10.1590/0034-7167-2020-0551

22. Pu J, Liu XX. Systematic perinatal management of the pregnant women and neonates during the epidemic of COVID-19. Chin J Obstet Gynecol 2020;55(3):153-6. doi: 10.3760/cma.j.cn112141-20200221-00123

23. Chen Z, Du LZ, Fu JF, Shu Q, Chen ZM, Shi LP, et al. Emergency plan for inter-hospital transfer of newborns with SARS-CoV-2 infection. Chin J Contemporary Pediatr 2020;22(3):226-30. doi: 10.7499/j.issn.1008-8830.2020.03.009

24. Working Group for the Prevention and Control of Neonatal 2019-nCoV Infection in the Perinatal Period of the Editorial Committee of Chinese Journal of Contemporary Pediatrics. Perinatal and neonatal management plan for prevention and control of 2019 novel coronavirus infection (1st ed). Chin J Contemporary Pediatr 2020;22(2):87-90. doi: 10.7499/j.issn.1008-8830.2020.02.001

25. Góes FGB, Santos AST, Lucchese I, Silva LJ, Silva LF, Silva MA. Boas práticas no cuidado ao recém-nascido em tempos de covid-19: revisão integrativa. Texto Contexto Enferm 2020;29:e20200242. doi: 10.1590/1980-265x-tce-2020-0242

26. Medical Association of Chinese People Liberation Army; Editorial Committee of Chinese Journal of Contemporary Pediatrics; Preparatory Group of Pediatric Disaster, Pediatric Society, Chinese Medical Association. Response plan in the neonatal intensive care unit during epidemic of SARS-CoV-2 infection (2nd Edition). Chin J Contemporary Pediatr 2020;22(3):205-10. doi: 10.7499/j.issn.1008-8830.2020.03.005 